\title{
CLASSIFICATION OF AIRBORNE LASER SCANNING DATA USING GEOMETRIC MULTI-SCALE FEATURES AND DIFFERENT NEIGHBOURHOOD TYPES
}

\author{
R. Blomley, B. Jutzi, M. Weinmann
}

Institute of Photogrammetry and Remote Sensing, Karlsruhe Institute of Technology (KIT)

Englerstr. 7, 76131 Karlsruhe, Germany - \{rosmarie.blomley, boris.jutzi, martin.weinmann\}@kit.edu

\author{
Commission III, WG III/2
}

KEY WORDS: ALS, LiDAR, Point Cloud, Features, Multi-Scale, Classification

\begin{abstract}
:
In this paper, we address the classification of airborne laser scanning data. We present a novel methodology relying on the use of complementary types of geometric features extracted from multiple local neighbourhoods of different scale and type. To demonstrate the performance of our methodology, we present results of a detailed evaluation on a standard benchmark dataset and we show that the consideration of multi-scale, multi-type neighbourhoods as the basis for feature extraction leads to improved classification results in comparison to single-scale neighbourhoods as well as in comparison to multi-scale neighbourhoods of the same type.
\end{abstract}

\section{INTRODUCTION}

Due to the increasing availability of laser scanning systems, more and more data is available in the form of 3D point clouds representing a point-wise sampling of physical object surfaces. Since a user-assisted analysis tends to be quite tedious and costly, an automated analysis of such 3D point clouds is desirable and hence, this automatic analysis has become an important task in photogrammetry, remote sensing and computer vision. In this context, particular interest has been paid to the detection of specific objects in the acquired data (Pu et al., 2011; Velizhev et al., 2012) and to the classification of each 3D point with respect to predefined class labels. For the latter, numerous approaches focus on classifying mobile laser scanning (MLS) data (Munoz et al., 2008; Munoz et al., 2009; Xiong et al., 2011; Hu et al., 2013; Bremer et al., 2013; Weinmann et al., 2015a; Weinmann et al., 2015c) or airborne laser scanning (ALS) data (Chehata et al., 2009; Shapovalov et al., 2010; Shapovalov and Velizhev, 2011; Mallet et al., 2011; Niemeyer et al., 2014; Xu et al., 2014; Guo et al., 2015). While MLS data provide a relatively dense and accurate sampling of the considered scene, a significantly lower point density and accuracy may be expected for ALS data. For both types of 3D point cloud data, however, the classification relies on descriptive features which allow an appropriate structural analysis and interpretation.

To analyse given 3D point clouds, a variety of approaches has been presented. However, a deeper analysis reveals that most of these approaches may be assigned to one of three major categories. Approaches of the first category focus on a fitting of geometric primitives (e.g. planes, cylinders or spheres) to the data and using the fit parameters as features (Vosselman et al., 2004). Instead of using such parametric features, the second category of approaches aims to describe the local context by evaluating certain geometric measures (e.g. shape measures represented by a single value which specifies one single property) based on the whole set of 3D points within the local neighbourhood (West et al., 2004; Mallet et al., 2011; Weinmann et al., 2013; Guo et al., 2015). These metrical features are to some degree interpretable as they describe fundamental properties of the local neighbourhood. The third category of approaches focuses on describing the local context by sampling the distribution of a certain metric (Osada et al., 2002; Rusu, 2009; Blomley et al., 2014), which results in distribution features. Meanwhile, especially metrical features and distribution features are widely but separately used for a variety of applications.

While geometric features characterizing the local 3D structure around a considered 3D point may be derived from a respective local neighbourhood, the consideration of multi-scale neighbourhoods additionally allows to consider the behaviour of the local 3D structure across neighbourhoods of different size. Combining features from multiple scales accounts for the characteristic scales of different structures and may hence be beneficial for classification (Brodu and Lague, 2012; Niemeyer et al., 2014; Schmidt et al., 2014; Hu et al., 2013). Yet, in the scope of classifying ALS data, multi-scale neighbourhoods are typically composed of local neighbourhoods of the same type (Niemeyer et al., 2014; Schmidt et al., 2014), while the consideration of different neighbourhood types in addition to different neighbourhood sizes may even provide a more promising alternative.

In this paper, we focus on a semantic interpretation of 3D point clouds covering a larger area and we therefore address the pointwise classification of ALS data. We investigate the combined use of metrical features and distribution features as well as the combined use of features from local neighbourhoods of different scale and type. We perform a thorough analysis of the features' and neighbourhoods' relative and combined performance. In summary, our main contributions consist in

- the extraction of complementary types of geometric features (represented by metrical features and distribution features),

- the use of different neighbourhood definitions (cylindrical, spherical) for feature extraction, and

- the consideration of metrical features and distribution features on multiple scales and different neighbourhood types.

After providing a brief review on related work in Section 2, we explain our methodology in Section 3. Subsequently, we focus on demonstrating the performance of this methodology. We therefore describe the involved data as well as the conducted experiments and present the respective results in Section 4. These results are discussed in detail in Section 5. Finally, in Section 6, we provide concluding remarks and suggestions for future work. 


\section{RELATED WORK}

To give an overview of related work, we focus on the different steps which have to be addressed for point cloud classification. First, a local neighbourhood has to be derived for each 3D point to be classified (Section 2.1). Subsequently, those 3D points within the respective local neighbourhood may be used to calculate geometric features (Section 2.2), which in turn provide the input for classification (Section 2.3).

\subsection{Neighbourhood Selection}

The local 3D structure around a considered 3D point $\mathbf{X}$ of a given $3 \mathrm{D}$ point cloud is contained in the spatial arrangement of other 3D points within the vicinity of $\mathbf{X}$. To characterise this local 3D structure, a suitable local neighbourhood has to be specified. In case a single neighbourhood definition is considered as the basis for feature extraction, the features derived provide a singlescale representation of the local 3D structure around X. However, features may also be derived from different local neighbourhoods in order to adequately describe $\mathbf{X}$, thus yielding a multi-scale representation. In the following, we summarise the main ideas for both alternatives.

2.1.1 Single-Scale Neighbourhoods: When defining the local neighbourhood for a given 3D point $\mathbf{X}$, the first step is to select an appropriate neighbourhood type. In this regard, the most commonly selected neighbourhood types are defined as follows:

- a spherical neighbourhood formed by all 3D points within a sphere around $\mathbf{X}$, which is parameterised with a fixed radius (Lee and Schenk, 2002),

- a cylindrical neighbourhood formed by all 3D points within a cylinder whose axis passes through $\mathbf{X}$ and whose radius is fixed (Filin and Pfeifer, 2005), or

- a neighbourhood formed by the $k \in \mathbb{N}$ nearest neighbours of X (Linsen and Prautzsch, 2001).

Note that all these neighbourhood types are parameterised by a single scale parameter (either a radius or $k$ ) which is typically selected to be identical for all points of the 3D point cloud. The selected value is derived via heuristic or empiric knowledge about the scene and/or the data. In this context, a smaller scale parameter allows to derive features describing fine details of the local 3D structure, whereas a larger scale parameter introduces a certain degree of smoothing and only allows to derive features coarsely describing the local 3D structure. Furthermore, the selection of an appropriate value depends on the sampling rate resulting from data acquisition as well as on the fact that suitable scales may depend on the objects of interest. In order to avoid the use of heuristic or empiric knowledge about the scene and/or the data, it has been proposed to derive a single, but optimal scale for each individual 3D point. Respective approaches mainly rely on the use of a local neighbourhood formed by the $k$ nearest neighbours of $\mathbf{X}$, while the locally optimal scale parameter $k_{\text {opt }}$ is derived via the consideration of the local surface variation (Pauly et al., 2003; Belton and Lichti, 2006), an iterative scheme based on curvature, point density and noise of normal estimation (Mitra and Nguyen, 2003; Lalonde et al., 2005), dimensionality-based scale selection (Demantké et al., 2011) or eigenentropy-based scale selection (Weinmann et al., 2015a; Weinmann, 2016).

2.1.2 Multi-Scale Neighbourhoods: While single-scale neighbourhoods allow to derive features which describe the local 3D structure at a specific scale, features derived from multi-scale neighbourhoods additionally allow to describe how the local 3D geometry behaves across scales (Brodu and Lague, 2012). Yet, respective approaches typically still involve heuristic or empiric knowledge about the scene and/or the data to select suitable values for the different scales (Brodu and Lague, 2012; Niemeyer et al., 2014; Schmidt et al., 2014). Furthermore, features may not only be extracted from one neighbourhood type with a varying scale parameter, but also from different neighbourhood types such as voxels, blocks and pillars (Hu et al., 2013). Again, however, these neighbourhood definitions typically involve heuristic or empiric knowledge about the scene and/or the data.

\subsection{Feature Extraction}

Once the neighbourhood has been determined for each 3D point, those $3 \mathrm{D}$ points within are considered in order to extract respective geometric features. In this context, options are either to extract $(i)$ interpretable features, whereby each feature is typically represented by a single value, or (ii) sampled features, whereby each feature is represented by a collection of values (e.g. in the form of histograms) and single values are hardly interpretable.

2.2.1 Interpretable Features: In order to obtain interpretable geometric features, the spatial arrangement of all points within the local neighbourhood of a respective $3 \mathrm{D}$ point $\mathbf{X}$ may be considered. Based on the $3 \mathrm{D}$ coordinates of all these $3 \mathrm{D}$ points, it is possible to calculate the $3 \mathrm{D}$ covariance matrix which is commonly referred to as the 3D structure tensor which preserves information about the local 3D structure. More specifically, the eigenvalues of the 3D structure tensor may (i) directly be exploited in order to distinguish between different shape primitives or (ii) be used in order to derive the covariance features of linearity $L_{\lambda}$, planarity $P_{\lambda}$, sphericity $S_{\lambda}$, omnivariance $O_{\lambda}$, anisotropy $A_{\lambda}$, eigenentropy $E_{\lambda}$ and sum of eigenvalues $\Sigma_{\lambda}$ (West et al., 2004) and local surface variation $C_{\lambda}$ (Pauly et al., 2003). The covariance features in particular have been involved in numerous investigations, and they have partially been combined with further geometric features, e.g. in (Munoz et al., 2008; Weinmann et al., 2013; Guo et al., 2015), or with full-waveform and echobased features, e.g. in (Chehata et al., 2009; Mallet et al., 2011; Niemeyer et al., 2012; Schmidt et al., 2014).

2.2.2 Sampled Features: One of the first approaches to extract sampled features from the local neighbourhood of a 3D point $\mathbf{X}$ has been presented in the form of the spin image descriptor (Johnson and Hebert, 1999), which results from spinning an image patch of predefined size around the surface normal vector at $\mathbf{X}$ and counting the number of 3D points falling in each pixel. Similarly, it is possible to divide a spherical neighbourhood centred at $\mathbf{X}$ into bins and (i) count the number of points in each bin as done with the 3D shape context descriptor (Frome et al., 2004) or (ii) sample a weighted histogram of normals as done with the Signature of Histograms of OrienTations (SHOT) descriptor (Tombari et al., 2010). A further approach has been presented with shape distributions (Osada et al., 2002), which sample point distances, angles, areas and volumes into histograms. While these shape distributions have originally been introduced to represent geometric properties of a complete 3D model (Osada et al., 2002), they have also been applied to describe 2.5D representations of objects (Wohlkinger and Vincze, 2011) or been adapted to characterise the local neighbourhood of a 3D point $\mathbf{X}$ that is part of a larger 3D point cloud (Blomley et al., 2014). A further sampling strategy has been proposed in the form of Point Feature Histograms (Rusu et al., 2008), which sample geometric relations between $3 \mathrm{D}$ points within the local neighbourhood of a $3 \mathrm{D}$ point $\mathbf{X}$ and the respective surface normal vectors into histograms. 


\subsection{Classification}

Once features have been derived, the next step is generally to uniquely assign a (semantic) class label to each $3 \mathrm{D}$ point of a given $3 \mathrm{D}$ point cloud. For this purpose, a supervised classification is commonly conducted, whereby the strategies of an individual or a contextual classification may be applied.

2.3.1 Individual Classification: The strategy of an individual classification exploits only the feature vector of each individual 3D point $\mathbf{X}$ to assign a (semantic) class label to this point. This assignment task has been tackled by focusing on very different learning principles and therefore involving very different classifiers such as a Maximum Likelihood classifier (Lalonde et al., 2005), an AdaBoost classifier (Lodha et al., 2007), a Random Forest (Chehata et al., 2009), a Support Vector Machine (Mallet et al., 2011) or a Bayesian Discriminant classifier (Khoshelham and Oude Elberink, 2012). While these approaches are relatively efficient and available in numerous software tools, the derived labelling can be of noisy appearance since no spatial correlation between labels of neighbouring 3D points is taken into account.

2.3.2 Contextual Classification: The strategy of a contextual classification employs the feature vector of the considered 3D point $\mathbf{X}$ as well as the spatial relationship to other neighbouring $3 \mathrm{D}$ points in order to assign the (semantic) class label. Among a variety of approaches used for 3D scene analysis are Associative Markov Networks (Munoz et al., 2008; Munoz et al., 2009), nonAssociative Markov Networks (Shapovalov et al., 2010; Shapovalov and Velizhev, 2011) and other inference procedures (Xiong et al., 2011; Shapovalov et al., 2013). Furthermore, it has been proposed to apply Conditional Random Fields on the basis of cylindrical neighbourhoods (Niemeyer et al., 2014; Schmidt et al., 2014) or on the basis of neighbourhoods formed by the $k$ nearest neighbours (Weinmann et al., 2015b). While such approaches tend to increase the computational burden, they result in a smooth labelling since interactions among neighbouring 3D points are taken into account.

\section{METHODOLOGY}

In this section, we present our novel methodology for point cloud classification which relies on geometric multi-scale features and different neighbourhood types. The main components of this methodology consist in (i) neighbourhood selection, (ii) feature extraction and (iii) classification, which are explained in the following subsections.

\subsection{Neighbourhood Selection}

As explained in Section 2.1, the first step of 3D scene analysis typically consists in defining appropriate local neighbourhoods as a basis for feature extraction. In this regard, we focus on both single-scale and multi-scale neighbourhoods, and we also consider different neighbourhood types as shown in Figure 1.

3.1.1 Single-Scale Neighbourhoods $\mathcal{N}_{\mathrm{c}, 1 \mathrm{~m}}, \mathcal{N}_{\mathrm{c}, 2 \mathrm{~m}}, \mathcal{N}_{\mathrm{c}, 3 \mathrm{~m}}$ and $\mathcal{N}_{\mathrm{c}, 5 \mathrm{~m}}$ : In accordance with other investigations focusing on the classification of airborne laser scanning data, we consider cylindrical neighbourhoods oriented in vertical direction. For the respective scale parameter, i.e. the radius of the cylinder, we use values of $1 \mathrm{~m}, 2 \mathrm{~m}, 3 \mathrm{~m}$ and $5 \mathrm{~m}$ as proposed in (Niemeyer et al., 2014; Schmidt et al., 2014) for classifying airborne laser scanning data and thus obtain the neighbourhoods $\mathcal{N}_{\mathrm{c}, 1 \mathrm{~m}}, \mathcal{N}_{\mathrm{c}, 2 \mathrm{~m}}, \mathcal{N}_{\mathrm{c}, 3 \mathrm{~m}}$ and $\mathcal{N}_{\mathrm{c}, 5 \mathrm{~m}}$.

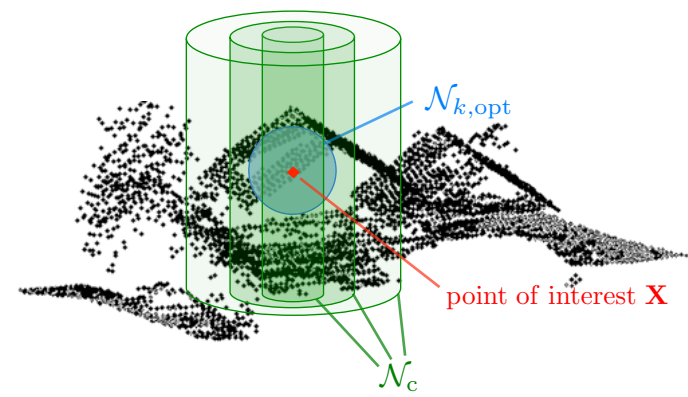

Figure 1. Concept sketch of neighbourhood definitions used in this work as basis for extracting features for a considered 3D point $\mathrm{X}$ : cylindrical neighbourhood definitions $\mathcal{N}_{\mathrm{c}}$ and the spherical neighbourhood $\mathcal{N}_{k, \text { opt }}$ formed by an optimal number $k_{\text {opt }}$ of nearest neighbours.

3.1.2 Single-Scale Neighbourhood $\mathcal{N}_{k \text {,opt }}$ : Furthermore, we intend to describe the local 3D structure of each considered 3D point $\mathbf{X}$ based on a local neighbourhood comprising $\mathbf{X}$ and its $k$ nearest neighbours. For this purpose, we involve spatially varying definitions of the local neighbourhood (i.e. varying values of the respective scale parameter $k$ ), which have proven to be favourable in comparison to identical neighbourhood definitions for all points of the 3D point cloud (Weinmann et al., 2015a; Weinmann et al., 2015b). Particularly eigenentropy-based scale selection (Weinmann et al., 2015a; Weinmann, 2016) has proven to increase the distinctiveness of the geometric features derived from those 3D points within the respective neighbourhood, and we therefore involve eigenentropy-based scale selection in our framework. Accordingly, we use the 3D coordinates of all points within the local neighbourhood in order to derive the 3D structure tensor and its eigenvalues. Subsequently, we normalise these eigenvalues by their sum which results in normalised eigenvalues $\lambda_{i}$ with $i \in\{1,2,3\}$ and these normalised eigenvalues, in turn, may be used to define the measure of eigenentropy according to

$$
E_{\lambda}=-\sum_{i=1}^{3} \lambda_{i} \ln \left(\lambda_{i}\right) .
$$

By representing this measure $E_{\lambda}$ as a function of the scale parameter $k$, the main idea of eigenentropy-based scale selection consists in minimizing $E_{\lambda}$ across varying values of $k$ which corresponds to minimizing the disorder of $3 \mathrm{D}$ points within the local neighbourhood and leads to the optimal scale parameter $k_{\text {opt }}$ for a considered $3 \mathrm{D}$ point $\mathbf{X}$ :

$$
k_{\mathrm{opt}}=\underset{k}{\arg \min } E_{\lambda}(k)
$$

For the involved scale parameter $k$, we consider all integer numbers within the interval $[10,100]$ as proposed in (Weinmann et al., 2015a; Weinmann et al., 2015c). The optimum-sized spherical neighbourhood $\mathcal{N}_{k \text {,opt }}$ is formed by the $k_{\text {opt }}$ nearest neighbours of $\mathbf{X}$.

3.1.3 Multi-Scale Neighbourhood $\mathcal{N}_{\mathrm{c} \text {,all }}$ ：To also account for the behaviour of the local 3D geometry across scales, we consider a multi-scale neighbourhood $\mathcal{N}_{\mathrm{c} \text {, all }}$ resulting from the combination of cylindrical neighbourhoods with radii of $1 \mathrm{~m}, 2 \mathrm{~m}, 3 \mathrm{~m}$ and $5 \mathrm{~m}$ which has also been proposed in (Niemeyer et al., 2014; Schmidt et al., 2014) for classifying airborne laser scanning data.

3.1.4 Multi-Scale, Multi-Type Neighbourhood $\mathcal{N}_{\text {all }}$ : We finally consider a multi-scale neighbourhood $\mathcal{N}_{\text {all }}$ resulting from the combination of the four cylindrical neighbourhoods $\mathcal{N}_{\mathrm{c}, 1 \mathrm{~m}}$, $\mathcal{N}_{\mathrm{c}, 2 \mathrm{~m}}, \mathcal{N}_{\mathrm{c}, 3 \mathrm{~m}}$ and $\mathcal{N}_{\mathrm{c}, 5 \mathrm{~m}}$ of varying radii and the spherical neighbourhood $\mathcal{N}_{k, \text { opt }}$ derived via eigenentropy-based scale selection. 


\subsection{Feature Extraction}

We intend to consider both interpretable features and sampled features, where the described neighbourhood definitions serve as a basis for feature extraction. Shape measures are chosen as interpretable features, since they are commonly involved for point cloud classification. For sampled features, we choose shape distributions, since the respective sampling is conducted independently from a local normal vector, which cannot always be estimated reliably for ALS data. Other alternatives such as the spin image descriptor, the 3D shape context descriptor, the SHOT descriptor and Point Feature Histograms require such a normal vector (cf. Section 2.2.2).

3.2.1 Shape Measures: To extract descriptive features for a 3D point $\mathbf{X}$ of a considered 3D point cloud, we first extract shape measures in terms of fundamental geometric properties as well as local 3D shape features characterizing the respectively considered local neighbourhood. Note that, in this context, we introduce the term of shape measures to describe features that comprise a single value each, whereby the value specifies one (mathematical) property of the whole set of 3D points within the evaluated local neighbourhood.

Among the fundamental geometric properties, we take into account a variety of geometric 3D properties (Weinmann et al. 2015a). These comprise the height $H=Z$ of the 3D point $\mathbf{X}$, the local point density $D$ derived from the number of 3D points within the local neighbourhood around $\mathbf{X}$, and the verticality $V$ represented by the $Z$-component of the eigenvector belonging to the smallest eigenvalue of the 3D structure tensor. Furthermore, we derive the maximum height difference $\Delta H$ and the standard deviation of height values $\sigma_{H}$ from all points within the respectively considered 3D neighbourhood. In case of spherical neighbourhoods whose scale parameter has been determined via eigenentropy-based scale selection, we additionally consider the radius $R$ of the considered local neighbourhood.

Among the local 3D shape features, we focus on the use of $\mathrm{co}$ variance features (West et al., 2004; Pauly et al., 2003) which are derived from the eigenvalues of the 3D structure tensor, where $\lambda_{1} \geq \lambda_{2} \geq \lambda_{3} \geq 0$ :

$$
\begin{aligned}
\text { Linearity: } & L_{\lambda}=\frac{\lambda_{1}-\lambda_{2}}{\lambda_{1}} \\
\text { Planarity: } & P_{\lambda}=\frac{\lambda_{2}-\lambda_{3}}{\lambda_{1}} \\
\text { Sphericity: } & S_{\lambda}=\frac{\lambda_{3}}{\lambda_{1}} \\
\text { Omnivariance: } & O_{\lambda}=\sqrt[3]{\prod_{i=1}^{3} \lambda_{i}} \\
\text { Anisotropy: } & A_{\lambda}=\frac{\lambda_{1}-\lambda_{3}}{\lambda_{1}} \\
\text { Eigenentropy: } & E_{\lambda}=-\sum_{i=1}^{3} \lambda_{i} \ln \left(\lambda_{i}\right) \\
\text { Sum of eigenvalues: } & \Sigma_{\lambda}=\sum_{i=1}^{3} \lambda_{i} \\
\text { Local surface variation: } & C_{\lambda}=\frac{\lambda_{3}}{\sum_{i=1}^{3} \lambda_{i}}
\end{aligned}
$$

3.2.2 Shape Distributions: Originally, shape distributions have been introduced as a parameterisation of overall object shape in (Osada et al., 2002). An adaptation of shape distributions as features for airborne laser scanning point cloud classification is given in (Blomley et al., 2014). In this paper, we follow the implementation of the aforementioned reference.

Shape distributions are histograms of shape values, which are derived from random point samples by applying five (distance or angular) metrics (cf. Figure 2). These metrics are:

- A3: the angle between any three random points,

- D1: the distance of one random point from the centroid of all points within the neighbourhood,

- D2: the distance between two random points,

- D3: the square root of the area spanned by a triangle between three random points, and

- D4: the cubic root of the volume spanned by a tetrahedron between four random points.

In order to use shape distributions as features in supervised classification, a fixed number of feature values has to be produced in a repeatable manner. Since the histogram counts of randomly sampled shape values within each local neighbourhood constitute the feature values, appropriate histogram binning thresholds and a matching (large enough) number of random pulls are crucial prerequisites. Following (Blomley et al., 2014), we choose 10 histogram bins, meaning that 10 feature values will be produced from each metric, and 255 as the number of pulls from the local neighbourhood. The binning thresholds of the histogram are estimated from the data prior to feature calculation in an adaptive histogram binning procedure. For this purpose, 500 exemplary local neighbourhoods are first evaluated in a fine-grained linear binning scope. Based on this fine-grained linearly-binned histogram, a transformation function to a non-linear binning scope with fewer bins is found in such a way that this large number of random samples produces even histogram count values.

\subsection{Feature Normalisation}

Based on the explanations on feature extraction, it becomes obvious that the extracted features address different quantities and therefore have different units as well as a different range of values. Accordingly, it is reasonable to introduce a normalisation allowing to span a feature space where each feature contributes approximately the same, independent of its unit and its range of values. Hence, we conduct a normalisation of both shape measures and shape distributions. For the analytic shape measures, a linear mapping to the interval between 0 and 1 is applied. To avoid the effect of outliers, the range of the data is determined by the 1st- and 99th-percentiles of the training data. Only if the absolute minimum is zero, the lower range value is set to zero too. For shape distributions, normalisation is achieved by dividing each histogram count by the total number of pulls from the local neighbourhood.

\subsection{Classification}

All features are concatenated to a feature vector and provided as input for classification. In the scope of this paper, we use a Random Forest classifier (Breiman, 2001) involving the principle of ensemble learning, where the aim is to strategically combine a set of weak learners to form a single strong learner. For such a combination, bagging (Breiman, 1996) represents a relatively intuitive way, since it focuses on training a weak learner of the same type for different subsets of the training data which are randomly drawn with replacement. Accordingly, the weak learners 


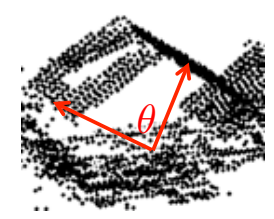

A3

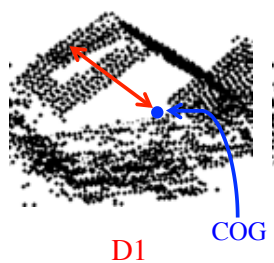

D1

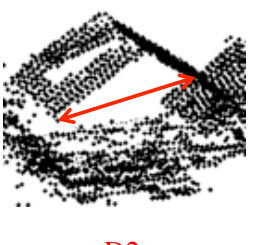

D2

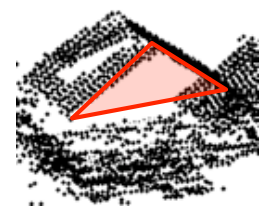

D3

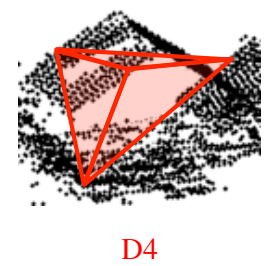

D4

Figure 2. Visualisation of the five shape distribution metrics A3, D1, D2, D3 and D4.

are all randomly different from each other and hence, taking the majority vote across the hypotheses of all weak learners results in a generalised and robust hypothesis of a single strong learner. When using decision trees as weak learners, the resulting strong learner represents a Random Forest classifier.

\section{EXPERIMENTAL RESULTS}

In this section, we provide details on the benchmark dataset used for performance evaluation (Section 4.1), describe the conducted experiments (Section 4.2) and present the results accomplished (Section 4.3).

\subsection{Dataset}

To examine the experimental performance of features from multiple scales and neighbourhood types for urban scene classification in airborne laser scanning data, we use a benchmark dataset presented in (Shapovalov et al., 2010). This dataset is kindly provided by the Graphics \& Media Lab, Moscow State University, and is publicly available ${ }^{1}$.

The dataset has been acquired with the airborne laser scanning system ALTM 2050 (Optech Inc.) and consists of two separate datasets (which are referred to as GML Dataset A and GML Dataset B), each of which is divided into a training and a testing part. For both GML Dataset A and GML Dataset B, a ground truth is available in the form of a point-wise labelling with respect to four semantic classes, namely ground, building, tree and low vegetation. The GML Dataset A additionally contains 3D points which are assigned to the class car. An overview of the number of labelled 3D points per class and dataset is given in Table 1.

\begin{tabular}{l|rr|rr} 
& \multicolumn{2}{|c}{ GML Dataset A } & \multicolumn{2}{c}{ GML Dataset B } \\
Class & Training & Testing & Training & Testing \\
\hline Ground & $557 \mathrm{k}$ & $440 \mathrm{k}$ & $1241 \mathrm{k}$ & $978 \mathrm{k}$ \\
Building & $98 \mathrm{k}$ & $20 \mathrm{k}$ & $148 \mathrm{k}$ & $55 \mathrm{k}$ \\
Car & $2 \mathrm{k}$ & $3 \mathrm{k}$ & - & - \\
Tree & $382 \mathrm{k}$ & $532 \mathrm{k}$ & $109 \mathrm{k}$ & $111 \mathrm{k}$ \\
Low vegetation & $35 \mathrm{k}$ & $8 \mathrm{k}$ & $47 \mathrm{k}$ & $17 \mathrm{k}$ \\
\hline$\Sigma$ & $1075 \mathrm{k}$ & $1003 \mathrm{k}$ & $1545 \mathrm{k}$ & $1161 \mathrm{k}$
\end{tabular}

Table 1. Number of labelled 3D points in the training set and in the test set for the two parts of the GML Dataset.

\subsection{Experiments}

For our experiments, we use different neighbourhood definitions as the basis for feature extraction: (i) cylindrical single-scale neighbourhoods defined by $\mathcal{N}_{\mathrm{c}, 1 \mathrm{~m}}, \mathcal{N}_{\mathrm{c}, 2 \mathrm{~m}}, \mathcal{N}_{\mathrm{c}, 3 \mathrm{~m}}$ and $\mathcal{N}_{\mathrm{c}, 5 \mathrm{~m}}$, (ii) a spherical single-scale neighbourhood defined by $\mathcal{N}_{k, \text { opt }}$, (iii) a multi-scale neighbourhood $\mathcal{N}_{\mathrm{c} \text {,all }}$ resulting from the combination of the cylindrical neighbourhoods $\mathcal{N}_{\mathrm{c}, 1 \mathrm{~m}}, \mathcal{N}_{\mathrm{c}, 2 \mathrm{~m}}, \mathcal{N}_{\mathrm{c}, 3 \mathrm{~m}}$ and $\mathcal{N}_{\mathrm{c}, 5 \mathrm{~m}}$, and (iv) a multi-scale neighbourhood $\mathcal{N}_{\text {all }}$ resulting from the combination of the neighbourhoods $\mathcal{N}_{\mathrm{c}, 1 \mathrm{~m}}, \mathcal{N}_{\mathrm{c}, 2 \mathrm{~m}}$, $\mathcal{N}_{\mathrm{c}, 3 \mathrm{~m}}, \mathcal{N}_{\mathrm{c}, 5 \mathrm{~m}}$ and $\mathcal{N}_{k, \text { opt }}$. The resulting features are concatenated to a feature vector and provided as input for a Random Forest, where we use the implementation available with (Liaw and

\footnotetext{
${ }^{1}$ http://graphics.cs.msu.ru/en/science/research/3dpoint/classification
}

\begin{tabular}{l|ccc|ccc} 
& \multicolumn{3}{|c}{ GML Dataset A } & \multicolumn{3}{c}{ GML Dataset B } \\
$\mathcal{N}$ & Shape & Shape & Both & Shape & Shape & Both \\
& Meas. & Distr. & & Meas. & Distr. & \\
\hline $\mathcal{N}_{\mathrm{c}, 1 \mathrm{~m}}$ & 25.21 & 34.09 & 35.72 & 31.87 & 32.46 & 36.44 \\
$\mathcal{N}_{\mathrm{c}, 2 \mathrm{~m}}$ & 30.61 & 42.35 & 44.48 & 32.39 & 35.84 & 37.21 \\
$\mathcal{N}_{\mathrm{c}, 3 \mathrm{~m}}$ & 32.24 & 44.76 & 48.64 & 31.59 & 33.39 & 35.10 \\
$\mathcal{N}_{\mathrm{c}, 5 \mathrm{~m}}$ & 41.64 & 42.05 & 50.42 & 27.59 & 27.43 & 28.87 \\
$\mathcal{N}_{k, \text { opt }}$ & 23.34 & 32.49 & 28.28 & 43.66 & 27.95 & 43.96 \\
$\mathcal{N}_{\mathrm{c}, \text { all }}$ & 35.45 & 48.37 & 49.61 & 35.52 & 38.20 & 39.54 \\
$\mathcal{N}_{\text {all }}$ & 57.28 & 53.93 & 61.17 & 59.70 & 58.31 & 63.76
\end{tabular}

Table 2. $C_{\kappa}$ (in \%) for different neighbourhood definitions and different feature sets.

\begin{tabular}{l|ccc|ccc} 
& \multicolumn{3}{|c}{ GML Dataset A } & \multicolumn{3}{c}{ GML Dataset B } \\
$\mathcal{N}$ & Shape & Shape & Both & Shape & Shape & Both \\
& Meas. & Distr. & & Meas. & Distr. & \\
\hline $\mathcal{N}_{\mathrm{c}, 1 \mathrm{~m}}$ & 50.22 & 56.62 & 57.88 & 63.65 & 65.57 & 68.60 \\
$\mathcal{N}_{\mathrm{c}, 2 \mathrm{~m}}$ & 55.49 & 64.07 & 65.68 & 64.07 & 68.76 & 69.05 \\
$\mathcal{N}_{\mathrm{c}, 3 \mathrm{~m}}$ & 57.70 & 66.14 & 69.12 & 62.92 & 66.05 & 66.61 \\
$\mathcal{N}_{\mathrm{c}, 5 \mathrm{~m}}$ & 65.10 & 63.88 & 70.76 & 58.09 & 59.16 & 59.44 \\
$\mathcal{N}_{k, \text { opt }}$ & 41.57 & 51.82 & 47.11 & 74.29 & 58.68 & 74.28 \\
$\mathcal{N}_{\text {c,all }}$ & 60.42 & 68.92 & 69.93 & 66.93 & 69.79 & 70.43 \\
$\mathcal{N}_{\text {all }}$ & 74.33 & 72.20 & 76.76 & 84.49 & 83.74 & 86.56
\end{tabular}

Table 3. OA (in \%) for different neighbourhood definitions and different feature sets.

Wiener, 2002). The number of trees $N_{T}$ of the Random Forest is determined via a standard grid search which focuses on testing different, heuristically selected values. Furthermore, we take into account that a training set with an unbalanced distribution of training examples per class tends to have a detrimental effect on the training process (Chen et al., 2004) and hence introduce a class re-balancing by randomly selecting an identical number of $N_{E}$ training examples per class to obtain a reduced training set. Thereby, $N_{E}=1000$ is considered to result in representative training data allowing to classify the considered classes.

First, we focus on a classification based on distinct feature groups (i.e. either shape measures or shape distributions) and, subsequently, we consider them in combination for the classification task. In order to compare the classification results obtained with the different approaches on point-level, we consider a variety of measures for evaluation on the respective test data: (i) Cohen's kappa $\left(C_{\kappa}\right)$, (ii) overall accuracy (OA), (iii) mean class recall (MCR) and (iv) mean class precision (MCP). Furthermore, we involve different measures for class-wise evaluation: (i) recall (REC), (ii) precision (PREC) and (iii) $F_{1}$-score.

\subsection{Results}

Due to the consideration of seven neighbourhood definitions, three different feature sets and two datasets, a total number of 42 experiments is conducted. The value of Cohen's kappa $C_{\kappa}$ and the overall accuracy (OA) for each of these experiments are provided in Table 2 and Table 3. Furthermore, we provide the respective values for mean class recall (MCR) and mean class precision (MCP) in Tables 4 and 5. To obtain an impression on the class-specific properties, the class-wise values for recall (REC) and precision (PREC) are given in Tables 6 and 7. Exemplary classification results are visualised in Figure 3.

General observations of the presented results reveal that the combination of shape measures and shape distributions produces im- 


\begin{tabular}{l|ccc|ccc} 
& \multicolumn{3}{|c}{ GML Dataset A } & \multicolumn{3}{c}{ GML Dataset B } \\
$\mathcal{N}$ & Shape & Shape & Both & Shape & Shape & Both \\
& Meas. & Distr. & & Meas. & Distr. & \\
\hline $\mathcal{N}_{\mathrm{c}, 1 \mathrm{~m}}$ & 45.90 & 52.96 & 52.73 & 72.73 & 69.57 & 75.14 \\
$\mathcal{N}_{\mathrm{c}, 2 \mathrm{~m}}$ & 49.53 & 58.77 & 57.97 & 72.53 & 71.73 & 76.40 \\
$\mathcal{N}_{\mathrm{c}, 3 \mathrm{~m}}$ & 49.34 & 59.47 & 60.32 & 71.96 & 69.76 & 74.94 \\
$\mathcal{N}_{\mathrm{c}, 5 \mathrm{~m}}$ & 46.50 & 56.57 & 58.17 & 69.06 & 64.43 & 70.66 \\
$\mathcal{N}_{k}$, opt & 36.42 & 47.44 & 39.19 & 74.99 & 64.33 & 75.70 \\
$\mathcal{N}_{\mathrm{c}, \text { all }}$ & 51.98 & 64.84 & 65.04 & 76.46 & 75.15 & 79.72 \\
$\mathcal{N}_{\text {all }}$ & 63.48 & 68.02 & 70.15 & 83.47 & 81.74 & 85.17
\end{tabular}

Table 4. MCR (in \%) for different neighbourhood definitions and different feature sets.

\begin{tabular}{l|ccc|ccc} 
& \multicolumn{3}{|c}{ GML Dataset A } & \multicolumn{3}{c}{ GML Dataset B } \\
$\mathcal{N}$ & Shape & Shape & Both & Shape & Shape & Both \\
& Meas. & Distr. & & Meas. & Distr. & \\
\hline $\mathcal{N}_{\mathrm{c}, 1 \mathrm{~m}}$ & 30.92 & 36.13 & 35.58 & 42.18 & 41.78 & 44.84 \\
$\mathcal{N}_{\mathrm{c}, 2 \mathrm{~m}}$ & 32.33 & 38.20 & 37.92 & 43.17 & 45.35 & 46.26 \\
$\mathcal{N}_{\mathrm{c}, 3 \mathrm{~m}}$ & 32.49 & 38.68 & 38.85 & 44.41 & 46.42 & 46.25 \\
$\mathcal{N}_{\mathrm{c}, 5 \mathrm{~m}}$ & 34.29 & 38.38 & 39.28 & 43.69 & 42.53 & 45.02 \\
$\mathcal{N}_{k, \text { opt }}$ & 35.81 & 37.50 & 37.12 & 50.96 & 49.65 & 51.72 \\
$\mathcal{N}_{\mathrm{c}, \text { all }}$ & 33.94 & 39.83 & 39.28 & 45.04 & 46.68 & 47.92 \\
$\mathcal{N}_{\text {all }}$ & 41.64 & 41.40 & 43.60 & 57.11 & 55.12 & 58.87
\end{tabular}

Table 5. MCP (in \%) for different neighbourhood definitions and different feature sets.

proved classification results compared to both separate groups. Furthermore, it can be observed that features extracted from multiscale neighbourhoods of the same type tend to lead to improved classification results. The combination of features derived from multi-scale, multi-type neighbourhoods does, in general, even lead to further improved classification results compared to features derived from multi-scale neighbourhoods of the same type.

A more detailed view on the derived results reveals that, when considering the evaluation among the single scales, there is no clear best neighbourhood scale among the cylindrical neighbourhoods $\mathcal{N}_{\mathrm{c}, 1 \mathrm{~m}}, \mathcal{N}_{\mathrm{c}, 2 \mathrm{~m}}, \mathcal{N}_{\mathrm{c}, 3 \mathrm{~m}}$ and $\mathcal{N}_{\mathrm{c}, 5 \mathrm{~m}}$. For shape measures, $\mathcal{N}_{\mathrm{c}, 5 \mathrm{~m}}$ (GML Dataset A) and $\mathcal{N}_{\mathrm{c}, 2 \mathrm{~m}}$ (GML Dataset B) perform well in class separability (Table 2 ) and in overall accuracy (Table 3). For shape distributions, $\mathcal{N}_{\mathrm{c}, 3 \mathrm{~m}}$ (GML Dataset A) and $\mathcal{N}_{\mathrm{c}, 2 \mathrm{~m}}$ (GML Dataset B) show the best results. The class-wise classification results reveal that different classes favour a different neighbourhood size (Tables 6 and 7). Furthermore, it may be stated that the spherical neighbourhood $\mathcal{N}_{k \text {,opt }}$, which is chosen via eigenentropy-based scale selection, behaves differently among the two datasets. On GML Dataset A, the resulting $C_{\kappa}$ shows that all classification results are below those of cylindrical neighbourhoods, while on GML Dataset B both the shape measures and the combined feature groups lead to superior results in comparison to the respective cylindrical neighbourhoods.

When considering features extracted from multiple scales, we may observe that the features extracted from multiple cylindrical neighbourhoods $\mathcal{N}_{\mathrm{c} \text {,all }}$ are usually similar to or slightly improved over the best classification result from the individual neighbourhoods (Tables 2 and 3). However, since there is a large variation among which neighbourhood size performs best in the two datasets, it seems worthwhile to test all scales.

When considering multi-scale, multi-type neighbourhoods, we may state that - even though the spherical neighbourhood selected via eigenentropy-based scale selection does not always perform very well on its own - there is usually a notable performance increase for the multi-type combination $\mathcal{N}_{\text {all }}$ over all other neighbourhood types or combinations.

\section{DISCUSSION}

The main focus throughout this work is to determine the behaviour of different feature types, namely shape measures as com-
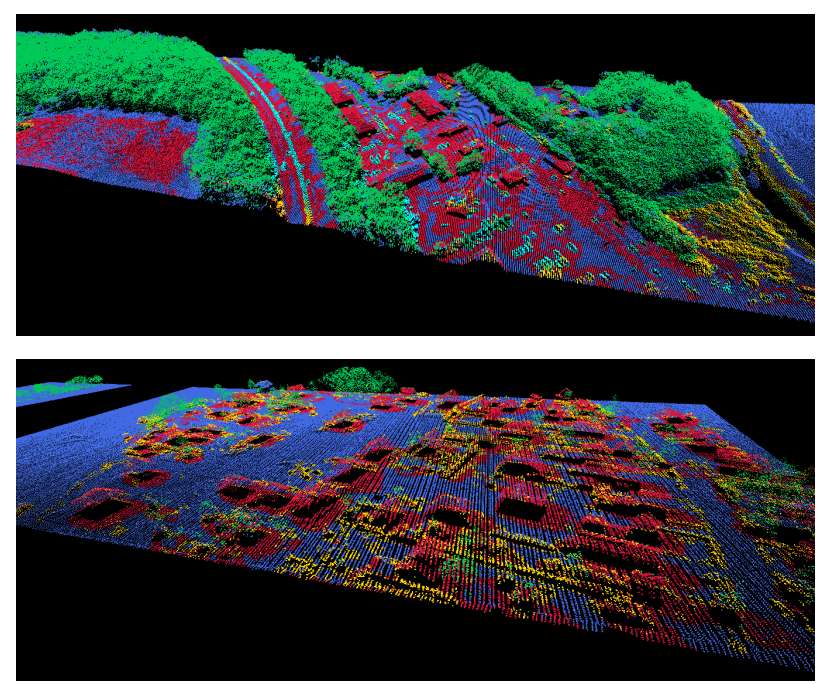

Figure 3. Visualisation of the classification results for GML Dataset A (top) and GML Dataset B (bottom) with the classes ground (blue), building (red), car (cyan), tree (green) and low vegetation (yellow).

mon representatives of interpretable features and shape distributions as an example of sampled features for neighbourhoods and neighbourhood combinations of different type and scale. This comparison has yielded the following insights.

The results accomplished here are comparable to those results of existing research. The most important possibility of comparison is to (Shapovalov et al., 2010), where the same two datasets have been used as well as a combination of metrical features and distribution features. While our methodology focuses on an improved characterisation of $3 \mathrm{D}$ points via feature extraction from local neighbourhoods of different scale and type, the methodology presented in (Shapovalov et al., 2010) focuses on the use of non-Associative Markov Networks and thus a contextual classification. As our approach only performs point-wise individual classification, we expect that not all results of the contextual classification may be matched. Comparing values of REC and PREC, we find that ground performs similar (on GML Dataset A, our inferior REC values are compensated for by a higher PREC, while on GML Dataset B only REC is slightly lower), buildings perform slightly better in REC, but worse in PREC on both datasets, car in GML Dataset A is detected with much higher REC, but lower PREC, tree is generally comparable (slightly lower REC on GML Dataset A and lower PREC but higher REC on GML Dataset B) and low vegetation again shows higher REC, but lower PREC values. Other qualitative comparisons may be sought for the individual feature groups. Overall, the performance is comparable and gives a positive evaluation of our results, considering that no contextual information is exploited.

Shape distributions have already been used in (Blomley et al., 2014) with cylindrical neighbourhoods for urban scene classification. There, the class-specific studies of classification performance across different cylinder radii indicated, that radii of $1-2 \mathrm{~m}$ are suitable for building and tree, while slightly larger radii of about $3 \mathrm{~m}$ are more suited for ground and low vegetation. A transfer of the class-wise REC and PREC values to $F_{1}$-scores for the single-scale neighbourhoods $\mathcal{N}_{\mathrm{c}, 1 \mathrm{~m}}, \mathcal{N}_{\mathrm{c}, 2 \mathrm{~m}}, \mathcal{N}_{\mathrm{c}, 3 \mathrm{~m}}$ and $\mathcal{N}_{\mathrm{c}, 5 \mathrm{~m}}$ shows best results for ground and low vegetation at radii of $2-3 \mathrm{~m}$ and best results for building and tree with 1-2m in GML Dataset $\mathrm{B}$, which matches the reference above, but best results for building and tree at radii of $3 \mathrm{~m}$ in GML Dataset $\mathrm{A}$, which does not agree with the reference above. 


\begin{tabular}{|c|c|c|c|c|c|c|}
\hline \multirow{3}{*}{$\mathcal{N}$} & \multicolumn{3}{|c|}{ GML Dataset A } & \multicolumn{3}{|c|}{ GML Dataset B } \\
\hline & Shape & Shape & Both & Shape & Shape & Both \\
\hline & Meas. & Distr. & & Meas. & Distr. & \\
\hline & \multicolumn{6}{|c|}{ Ground } \\
\hline $\mathcal{N}_{\mathrm{c}, 1 \mathrm{~m}}$ & 50.82 & 34.01 & 43.17 & 60.35 & 63.41 & 66.10 \\
\hline $\mathcal{N}_{\mathrm{c}, 2 \mathrm{~m}}$ & 51.30 & 40.25 & 46.14 & 60.78 & 66.95 & 66.34 \\
\hline $\mathcal{N}_{\mathrm{c}, 3 \mathrm{~m}}$ & 55.42 & 42.47 & 49.63 & 59.29 & 63.66 & 63.29 \\
\hline $\mathcal{N}_{\mathrm{c}, 5 \mathrm{~m}}$ & 57.05 & 37.51 & 48.92 & 54.05 & 56.24 & 55.41 \\
\hline $\mathcal{N}_{k, \text { opt }}$ & 43.12 & 40.07 & 44.36 & 72.93 & 55.16 & 72.66 \\
\hline $\mathcal{N}_{\mathrm{c}, \text { all }}$ & 57.13 & 46.38 & 55.63 & 63.51 & 67.00 & 67.17 \\
\hline \multirow[t]{2}{*}{$\mathcal{N}_{\text {all }}$} & 64.57 & 52.38 & 62.89 & 83.82 & 82.93 & 86.02 \\
\hline & \multicolumn{6}{|c|}{ Building } \\
\hline $\mathcal{N}_{\mathrm{c}, 1 \mathrm{~m}}$ & 50.79 & 43.19 & 50.61 & 69.51 & 54.98 & 69.55 \\
\hline $\mathcal{N}_{\mathrm{c}, 2 \mathrm{~m}}$ & 50.75 & 42.52 & 48.63 & 71.04 & 61.54 & 75.55 \\
\hline $\mathcal{N}_{\mathrm{c}, 3 \mathrm{~m}}$ & 53.18 & 38.26 & 48.14 & 73.18 & 56.51 & 75.37 \\
\hline $\mathcal{N}_{\mathrm{c}, 5 \mathrm{~m}}$ & 56.38 & 39.33 & 53.75 & 71.81 & 52.27 & 68.61 \\
\hline $\mathcal{N}_{k, \text { opt }}$ & 62.85 & 48.89 & 60.11 & 66.93 & 52.11 & 68.61 \\
\hline $\mathcal{N}_{\mathrm{c}, \text { all }}$ & 60.47 & 45.70 & 53.78 & 80.74 & 74.05 & 84.34 \\
\hline \multirow[t]{2}{*}{$\mathcal{N}_{\text {all }}$} & 63.82 & 53.58 & 64.81 & 80.58 & 80.01 & 84.00 \\
\hline & \multicolumn{6}{|c|}{ Car } \\
\hline $\mathcal{N}_{\mathrm{c}, 1 \mathrm{~m}}$ & 59.97 & 62.04 & 69.03 & - & - & - \\
\hline $\mathcal{N}_{\mathrm{c}, 2 \mathrm{~m}}$ & 56.51 & 66.77 & 68.22 & - & - & - \\
\hline $\mathcal{N}_{\mathrm{c}, 3 \mathrm{~m}}$ & 49.92 & 63.31 & 62.47 & - & - & - \\
\hline $\mathcal{N}_{\mathrm{c}, 5 \mathrm{~m}}$ & 18.86 & 52.46 & 38.55 & - & - & - \\
\hline $\mathcal{N}_{k, \text { opt }}$ & 33.35 & 45.81 & 31.56 & - & - & - \\
\hline $\mathcal{N}_{\mathrm{c}, \text { all }}$ & 37.56 & 73.45 & 71.59 & - & - & - \\
\hline \multirow[t]{2}{*}{$\mathcal{N}_{\text {all }}$} & 45.19 & 74.31 & 71.00 & - & - & - \\
\hline & \multicolumn{6}{|c|}{ Tree } \\
\hline $\mathcal{N}_{\mathrm{c}, 1 \mathrm{~m}}$ & 50.11 & 75.90 & 70.64 & 88.46 & 88.97 & 89.11 \\
\hline $\mathcal{N}_{\mathrm{c}, 2 \mathrm{~m}}$ & 59.50 & 84.61 & 82.77 & 88.79 & 87.98 & 88.79 \\
\hline $\mathcal{N}_{\mathrm{c}, 3 \mathrm{~m}}$ & 60.23 & 86.75 & 86.27 & 89.40 & 91.57 & 91.06 \\
\hline $\mathcal{N}_{\mathrm{c}, 5 \mathrm{~m}}$ & 72.91 & 86.62 & 89.80 & 85.95 & 87.95 & 88.96 \\
\hline $\mathcal{N}_{k, \text { opt }}$ & 40.13 & 61.85 & 49.53 & 90.60 & 93.26 & 92.06 \\
\hline $\mathcal{N}_{c, \text { all }}$ & 63.56 & 88.38 & 82.47 & 89.53 & 92.71 & 91.53 \\
\hline \multirow[t]{2}{*}{$\mathcal{N}_{\text {all }}$} & 83.17 & 89.30 & 88.92 & 93.65 & 94.92 & 94.10 \\
\hline & \multicolumn{6}{|c|}{ Low vegetation } \\
\hline $\mathcal{N}_{\mathrm{c}, 1 \mathrm{~m}}$ & 17.83 & 49.68 & 30.20 & 72.59 & 70.93 & 75.82 \\
\hline $\mathcal{N}_{\mathrm{c}, 2 \mathrm{~m}}$ & 29.58 & 59.67 & 44.11 & 69.52 & 70.43 & 74.94 \\
\hline $\mathcal{N}_{\mathrm{c}, 3 \mathrm{~m}}$ & 27.96 & 66.56 & 55.07 & 65.97 & 67.31 & 70.06 \\
\hline $\mathcal{N}_{\mathrm{c}, 5 \mathrm{~m}}$ & 27.31 & 66.90 & 59.85 & 64.44 & 61.26 & 69.67 \\
\hline $\mathcal{N}_{k, \text { opt }}$ & 2.63 & 40.58 & 10.40 & 69.49 & 56.78 & 69.45 \\
\hline $\mathcal{N}_{\mathrm{c}, \text { all }}$ & 41.18 & 70.28 & 61.70 & 72.05 & 66.85 & 75.86 \\
\hline $\mathcal{N}_{\text {all }}$ & 60.65 & 70.52 & 63.11 & 75.83 & 69.11 & 76.57 \\
\hline
\end{tabular}

Table 6. Class-wise REC (in \%) for different neighbourhood definitions and different feature sets.

A comparison of the classification results derived for cylindrical single-scale neighbourhoods and multi-scale neighbourhoods of the same (cylindrical) type reveals that the behaviour of the local 3D structure across different scales provides information which is relevant for the classification task. This becomes visible in improved classification results for multi-scale neighbourhoods of the same type. Furthermore, we may state that the different neighbourhood types capture complementary information about the local 3D structure. This clearly becomes visible in the improved classification results obtained for multi-scale, multi-type neighbourhoods in comparison to multi-scale neighbourhoods of the same type. Despite the weak performance of the $\mathcal{N}_{k \text {,opt }}$ neighbourhood (which has originally been developed for MLS data) on its own, its combination with the cylindrical neighbourhoods provides a significant improvement over the result obtained when considering all cylindrical neighbourhoods $\mathcal{N}_{\mathrm{c} \text {,all }}$.

\section{CONCLUSIONS AND FUTURE WORK}

In this paper, we have presented a methodology for classifying airborne laser scanning data. The novelty of this methodology consists in the use of complementary types of geometric features extracted from multiple scales and different neighbourhood types. In a detailed evaluation, we have demonstrated that the consideration of multi-scale, multi-type neighbourhoods as the basis for feature extraction leads to improved classification results in comparison to single-scale neighbourhoods as well as in comparison to multi-scale neighbourhoods of the same type. Accordingly, we may state that multi-scale, multi-type neighbourhoods are well-suited for point cloud classification, which may

\begin{tabular}{|c|c|c|c|c|c|c|}
\hline \multirow{3}{*}{$\mathcal{N}$} & \multicolumn{3}{|c|}{ GML Dataset A } & \multicolumn{3}{|c|}{ GML Dataset B } \\
\hline & Shape & Shape & Both & Shape & Shape & Both \\
\hline & Meas. & Distr. & & Meas. & Distr. & \\
\hline & \multicolumn{6}{|c|}{ Ground } \\
\hline $\mathcal{N}_{\text {c }, 1 \mathrm{~m}}$ & 62.52 & 87.51 & 82.76 & 99.22 & 98.41 & 98.96 \\
\hline $\mathcal{N}_{\mathrm{c}, 2 \mathrm{~m}}$ & 64.02 & 92.38 & 88.89 & 99.43 & 98.84 & 99.21 \\
\hline $\mathcal{N}_{\mathrm{c}, 3 \mathrm{~m}}$ & 62.77 & 93.48 & 89.93 & 99.56 & 99.04 & 99.51 \\
\hline $\mathcal{N}_{\mathrm{c}, 5 \mathrm{~m}}$ & 70.72 & 94.30 & 91.11 & 99.37 & 99.23 & 99.62 \\
\hline $\mathcal{N}_{k, \text { opt }}$ & 91.52 & 89.08 & 93.31 & 99.14 & 97.47 & 99.31 \\
\hline $\mathcal{N}_{\mathrm{c}, \text { all }}$ & 63.57 & 93.99 & 82.37 & 99.40 & 99.40 & 99.59 \\
\hline \multirow[t]{2}{*}{$\mathcal{N}_{\text {all }}$} & 85.75 & 95.07 & 93.90 & 99.58 & 99.62 & 99.78 \\
\hline & \multicolumn{6}{|c|}{ Building } \\
\hline $\mathcal{N}_{\mathrm{c}, 1 \mathrm{~m}}$ & 6.79 & 5.34 & 6.79 & 24.81 & 21.90 & 30.80 \\
\hline $\mathcal{N}_{\mathrm{c}, 2 \mathrm{~m}}$ & 6.50 & 6.25 & 7.40 & 32.84 & 37.34 & 38.20 \\
\hline $\mathcal{N}_{\mathrm{c}, 3 \mathrm{~m}}$ & 7.96 & 6.67 & 8.95 & 41.31 & 47.72 & 44.08 \\
\hline $\mathcal{N}_{\mathrm{c}, 5 \mathrm{~m}}$ & 10.16 & 7.50 & 10.82 & 42.18 & 37.58 & 46.12 \\
\hline $\mathcal{N}_{k, \text { opt }}$ & 7.11 & 5.74 & 6.97 & 16.54 & 9.19 & 16.05 \\
\hline $\mathcal{N}_{\mathrm{c}, \text { all }}$ & 9.71 & 8.13 & 9.97 & 35.57 & 38.43 & 41.33 \\
\hline \multirow[t]{2}{*}{$\mathcal{N}_{\text {all }}$} & 11.63 & 9.55 & 11.62 & 33.33 & 33.70 & 38.54 \\
\hline & \multicolumn{6}{|c|}{ Car } \\
\hline $\mathcal{N}_{\mathrm{c}, 1 \mathrm{~m}}$ & 2.83 & 3.07 & 3.43 & - & - & - \\
\hline $\mathcal{N}_{\text {c }, 2 \mathrm{~m}}$ & 4.67 & 3.87 & 5.19 & - & - & - \\
\hline $\mathcal{N}_{\mathrm{c}, 3 \mathrm{~m}}$ & 4.67 & 3.31 & 5.46 & - & - & - \\
\hline $\mathcal{N}_{\mathrm{c}, 5 \mathrm{~m}}$ & 2.33 & 2.37 & 4.09 & - & - & - \\
\hline $\mathcal{N}_{k, \text { opt }}$ & 0.62 & 1.18 & 0.78 & - & - & - \\
\hline $\mathcal{N}_{\mathrm{c}, \text { all }}$ & 6.90 & 6.61 & 11.18 & - & - & - \\
\hline \multirow[t]{2}{*}{$\mathcal{N}_{\text {all }}$} & 11.46 & 7.75 & 12.80 & - & - & - \\
\hline & \multicolumn{6}{|c|}{ Tree } \\
\hline $\mathcal{N}_{\mathrm{c}, 1 \mathrm{~m}}$ & 81.09 & 81.27 & 82.76 & 29.69 & 31.23 & 31.64 \\
\hline $\mathcal{N}_{\mathrm{c}, 2 \mathrm{~m}}$ & 83.56 & 82.29 & 83.63 & 28.56 & 29.34 & 30.30 \\
\hline $\mathcal{N}_{\mathrm{c}, 3 \mathrm{~m}}$ & 83.73 & 82.36 & 83.80 & 27.67 & 28.10 & 28.82 \\
\hline $\mathcal{N}_{\mathrm{c}, 5 \mathrm{~m}}$ & 84.13 & 82.06 & 83.10 & 27.37 & 27.29 & 27.73 \\
\hline $\mathcal{N}_{k, \text { opt }}$ & 79.71 & 89.31 & 84.11 & 74.28 & 86.53 & 76.42 \\
\hline $\mathcal{N}_{\mathrm{c}, \text { all }}$ & 84.89 & 82.87 & 85.81 & 29.97 & 30.89 & 31.46 \\
\hline \multirow[t]{2}{*}{$\mathcal{N}_{\text {all }}$} & 93.17 & 87.38 & 92.75 & 77.51 & 68.70 & 75.76 \\
\hline & \multicolumn{6}{|c|}{ Low vegetation } \\
\hline $\mathcal{N}_{\mathrm{c}, 1 \mathrm{~m}}$ & 1.37 & 3.48 & 2.17 & 14.98 & 15.58 & 17.98 \\
\hline $\mathcal{N}_{\mathrm{c}, 2 \mathrm{~m}}$ & 2.90 & 6.19 & 4.48 & 11.85 & 15.86 & 17.33 \\
\hline $\mathcal{N}_{\mathrm{c}, 3 \mathrm{~m}}$ & 3.29 & 7.59 & 6.14 & 9.11 & 10.82 & 12.57 \\
\hline $\mathcal{N}_{\mathrm{c}, 5 \mathrm{~m}}$ & 4.11 & 5.66 & 7.30 & 5.84 & 6.02 & 6.59 \\
\hline $\mathcal{N}_{k, \text { opt }}$ & 0.11 & 2.19 & 0.45 & 13.87 & 5.40 & 15.09 \\
\hline $\mathcal{N}_{\mathrm{c}, \text { all }}$ & 4.62 & 7.57 & 7.08 & 15.22 & 17.99 & 19.30 \\
\hline $\mathcal{N}_{\text {all }}$ & 6.19 & 7.25 & 6.93 & 18.02 & 18.46 & 21.41 \\
\hline
\end{tabular}

Table 7. Class-wise PREC (in \%) for different neighbourhood definitions and different feature sets.

be motivated by the fact that they not only allow to describe the local 3D structure at each considered 3D point, but also the behaviour of the local 3D structure across scales and the behaviour of the local 3D structure across different neighbourhood types.

In future work, we plan to extend the presented methodology by additionally considering contextual information inherent in the data in order to further improve the classification results. Besides such an extension, it would also be desirable to adapt the presented methodology to different types of point cloud data (e.g. terrestrial or mobile laser scanning data which provide a dense sampling) and/or to use the derived classification results as the basis for a subsequent extraction of objects of interest. This, in turn, might represent an important prerequisite for tasks relying on the results of object-based scene analysis, e.g. for city modelling in terms of deriving an abstraction of the acquired point cloud data or for urban accessibility analysis in terms of navigating people in wheelchairs through complex urban environments. Furthermore, we aim to address a transfer of the presented concepts to vegetation analysis which represents a promising field of application for multi-scale approaches.

\section{ACKNOWLEDGEMENTS}

This work was partially supported by the Carl-Zeiss foundation [Nachwuchsförderprogramm 2014].

\section{REFERENCES}

Belton, D. and Lichti, D. D., 2006. Classification and segmentation of terrestrial laser scanner point clouds using local variance information. The 
International Archives of the Photogrammetry, Remote Sensing and Spatial Information Sciences, Vol. XXXVI-5, pp. 44-49.

Blomley, R., Weinmann, M., Leitloff, J. and Jutzi, B., 2014. Shape distribution features for point cloud analysis - A geometric histogram approach on multiple scales. ISPRS Annals of the Photogrammetry, Remote Sensing and Spatial Information Sciences, Vol. II-3, pp. 9-16.

Breiman, L., 1996. Bagging predictors. Machine Learning, 24(2) pp. 123-140.

Breiman, L., 2001. Random forests. Machine Learning, 45(1), pp. 5-32. Bremer, M., Wichmann, V. and Rutzinger, M., 2013. Eigenvalue and graph-based object extraction from mobile laser scanning point clouds ISPRS Annals of the Photogrammetry, Remote Sensing and Spatial Information Sciences, Vol. II-5/W2, pp. 55-60.

Brodu, N. and Lague, D., 2012. 3D terrestrial lidar data classification of complex natural scenes using a multi-scale dimensionality criterion applications in geomorphology. ISPRS Journal of Photogrammetry and Remote Sensing, 68, pp. 121-134.

Chehata, N., Guo, L. and Mallet, C., 2009. Airborne lidar feature selection for urban classification using random forests. The International Archives of the Photogrammetry, Remote Sensing and Spatial Information Sciences, Vol. XXXVIII-3/W8, pp. 207-212.

Chen, C., Liaw, A. and Breiman, L., 2004. Using random forest to learn imbalanced data. Technical Report, University of California, Berkeley, USA.

Demantké, J., Mallet, C., David, N and Vallet, B., 2011. Dimensionality based scale selection in 3D lidar point clouds. The International Archives of the Photogrammetry, Remote Sensing and Spatial Information Sciences, Vol. XXXVIII-5/W12, pp. 97-102.

Filin, S. and Pfeifer, N., 2005. Neighborhood systems for airborne lase data. Photogrammetric Engineering \& Remote Sensing, 71(6), pp. 743 755.

Frome, A., Huber, D., Kolluri, R., Bülow, T. and Malik, J., 2004. Recognizing objects in range data using regional point descriptors. Proceeding of the European Conference on Computer Vision, Vol. III, pp. 224-237.

Guo, B., Huang, X., Zhang, F. and Sohn, G., 2015. Classification of airborne laser scanning data using JointBoost. ISPRS Journal of Photogrammetry and Remote Sensing, 100, pp. 71-83.

Hu, H., Munoz, D., Bagnell, J. A. and Hebert, M., 2013. Efficient 3-D scene analysis from streaming data. Proceedings of the IEEE International Conference on Robotics and Automation, pp. 2297-2304.

Johnson, A. E. and Hebert, M., 1999. Using spin images for efficient object recognition in cluttered 3D scenes. IEEE Transactions on Pattern Analysis and Machine Intelligence, 21(5), pp. 433-449.

Khoshelham, K. and Oude Elberink, S. J., 2012. Role of dimensionality reduction in segment-based classification of damaged building roofs in airborne laser scanning data. Proceedings of the International Conference on Geographic Object Based Image Analysis, pp. 372-377.

Lalonde, J.-F., Unnikrishnan, R., Vandapel, N. and Hebert, M., 2005. Scale selection for classification of point-sampled 3D surfaces. Proceed ings of the International Conference on 3-D Digital Imaging and Modeling, pp. 285-292.

Lee, I. and Schenk, T., 2002. Perceptual organization of 3D surface points. The International Archives of the Photogrammetry. Remote Sensing and Spatial Information Sciences, Vol. XXXIV-3A, pp. 193-198.

Liaw, A. and Wiener, M., 2002. Classification and regression by randomForest. R News, Vol. 2/3, pp. 18-22.

Linsen, L. and Prautzsch, H., 2001. Local versus global triangulations. Proceedings of Eurographics, pp. 257-263.

Lodha, S. K., Fitzpatrick, D. M. and Helmbold, D. P., 2007. Aerial lidar data classification using AdaBoost. Proceedings of the International Conference on 3-D Digital Imaging and Modeling, pp. 435-442.

Mallet, C., Bretar, F., Roux, M., Soergel, U. and Heipke, C., 2011. Relevance assessment of full-waveform lidar data for urban area classification. ISPRS Journal of Photogrammetry and Remote Sensing, 66(6), pp. S71S84.

Mitra, N. J. and Nguyen, A., 2003. Estimating surface normals in noisy point cloud data. Proceedings of the Annual Symposium on Computational Geometry, pp. 322-328.

Munoz, D., Bagnell, J. A., Vandapel, N. and Hebert, M., 2009. Contextual classification with functional max-margin Markov networks. Proceedings of the IEEE Conference on Computer Vision and Pattern Recognition, pp. 975-982.

Munoz, D., Vandapel, N. and Hebert, M., 2008. Directional associative Markov network for 3-D point cloud classification. Proceedings of the International Symposium on 3D Data Processing, Visualization and Transmission, pp. 63-70.
Niemeyer, J., Rottensteiner, F. and Soergel, U., 2012. Conditional random fields for lidar point cloud classification in complex urban areas. ISPRS Annals of the Photogrammetry, Remote Sensing and Spatial Information Sciences, Vol. I-3, pp. 263-268.

Niemeyer, J., Rottensteiner, F. and Soergel, U., 2014. Contextual classification of lidar data and building object detection in urban areas. ISPRS Journal of Photogrammetry and Remote Sensing, 87, pp. 152-165.

Osada, R., Funkhouser, T., Chazelle, B. and Dobkin, D., 2002. Shape distributions. ACM Transactions on Graphics, 21(4), pp. 807-832.

Pauly, M., Keiser, R. and Gross, M., 2003. Multi-scale feature extraction on point-sampled surfaces. Computer Graphics Forum, 22(3), pp. 81-89. Pu, S., Rutzinger, M., Vosselman, G. and Oude Elberink, S., 2011. Recognizing basic structures from mobile laser scanning data for road inventory studies. ISPRS Journal of Photogrammetry and Remote Sensing, 66(6), pp. S28-S39.

Rusu, R. B., 2009. Semantic 3D object maps for everyday manipulation in human living environments. PhD thesis, Computer Science department, Technische Universität München, Germany.

Rusu, R. B., Marton, Z. C., Blodow, N. and Beetz, M., 2008. Persistent point feature histograms for $3 \mathrm{D}$ point clouds. Proceedings of the International Conference on Intelligent Autonomous Systems, pp. 119-128.

Schmidt, A., Niemeyer, J., Rottensteiner, F. and Soergel, U., 2014. Contextual classification of full waveform lidar data in the Wadden Sea. IEEE Geoscience and Remote Sensing Letters, 11(9), pp. 1614-1618.

Shapovalov, R. and Velizhev, A., 2011. Cutting-plane training of nonassociative Markov network for 3D point cloud segmentation. Proceed ings of the IEEE International Conference on 3D Digital Imaging, Modeling, Processing, Visualization and Transmission, pp. 1-8.

Shapovalov, R., Velizhev, A. and Barinova, O., 2010. Non-associative Markov networks for 3D point cloud classification. The International Archives of the Photogrammetry, Remote Sensing and Spatial Information Sciences, Vol. XXXVIII-3A, pp. 103-108.

Shapovalov, R., Vetrov, D. and Kohli, P., 2013. Spatial inference machines. Proceedings of the IEEE Conference on Computer Vision and Pattern Recognition, pp. 2985-2992.

Tombari, F., Salti, S. and Di Stefano, L., 2010. Unique signatures of histograms for local surface description. Proceedings of the European Conference on Computer Vision, Vol. III, pp. 356-369.

Velizhev, A., Shapovalov, R. and Schindler, K., 2012. Implicit shape models for object detection in 3D point clouds. ISPRS Annals of the Photogrammetry, Remote Sensing and Spatial Information Sciences, Vol. I-3, pp. 179-184.

Vosselman, G., Gorte, B. G. H., Sithole, G. and Rabbani, T., 2004. Recognising structure in laser scanner point clouds. The International Archives of the Photogrammetry, Remote Sensing and Spatial Information Sciences, Vol. XXXVI-8/W2, pp. 33-38.

Weinmann, M., 2016. Reconstruction and analysis of $3 D$ scenes - From irregularly distributed $3 D$ points to object classes. Springer, Cham, Switzerland.

Weinmann, M., Jutzi, B. and Mallet, C., 2013. Feature relevance assessment for the semantic interpretation of 3D point cloud data. ISPRS Annals of the Photogrammetry, Remote Sensing and Spatial Information Sciences, Vol. II-5/W2, pp. 313-318.

Weinmann, M., Jutzi, B., Hinz, S. and Mallet, C., 2015a. Semantic point cloud interpretation based on optimal neighborhoods, relevant features and efficient classifiers. ISPRS Journal of Photogrammetry and Remote Sensing, 105, pp. 286-304.

Weinmann, M., Schmidt, A., Mallet, C., Hinz, S., Rottensteiner, F. and Jutzi, B., 2015b. Contextual classification of point cloud data by exploiting individual 3D neighborhoods. ISPRS Annals of the Photogrammetry Remote Sensing and Spatial Information Sciences, Vol. II-3/W4, pp. 271278.

Weinmann, M. Urban, S., Hinz, S., Jutzi, B. and Mallet, C., 2015c. Distinctive 2D and 3D features for automated large-scale scene analysis in urban areas. Computers \& Graphics, 49, pp. 47-57.

West, K. F., Webb, B. N., Lersch, J. R., Pothier, S., Triscari, J. M. and Iverson, A. E., 2004. Context-driven automated target detection in 3-D data. Proceedings of SPIE, Vol. 5426, pp. 133-143.

Wohlkinger, W. and Vincze, M., 2011. Ensemble of shape functions for 3D object classification. Proceedings of the IEEE International Confer ence on Robotics and Biomimetics, pp. 2987-2992.

Xiong, X., Munoz, D., Bagnell, J. A. and Hebert, M., 2011. 3-D scene analysis via sequenced predictions over points and regions. Proceed ings of the IEEE International Conference on Robotics and Automation, pp. 2609-2616.

Xu, S., Vosselman, G. and Oude Elberink, S., 2014. Multiple-entity based classification of airborne laser scanning data in urban areas. ISPRS Journal of Photogrammetry and Remote Sensing, 88, pp. 1-15. 